LAUR-95-950

\title{
The effect of inelastic processes on tunneling
}

\author{
Janez Bonča ${ }^{a, b, c}$ and S. A. Trugman ${ }^{a}$ \\ ${ }^{a}$ Theory Division and ${ }^{b}$ Center for Nonlinear Studies, Los Alamos National Laboratory, Los \\ Alamos, NM 87545, \\ ${ }^{c}$ J. Stefan Institute, University of Ljubljana, 61111, Slovenia
}

(June 23, 2021)

\begin{abstract}
We study an electron that interacts with phonons or other linear or nonlinear excitations as it resonantly tunnels. The method we use is based on mapping a many-body problem in a large variational space exactly onto a onebody problem. The method is conceptually simpler than previous Green's function approaches, and allows the essentially exact numerical solution of much more general problems. We solve tunneling problems with transverse channels, multiple sites coupled to phonons, and multiple phonon degrees of freedom and excitations.
\end{abstract}

PACS-73.40.Gk, 71.38.+i, 73.50.Fq, 73.50.Bk

Typeset using REVTEX 
We consider a single electron tunneling through a resonant tunneling diode or a quantum dot, in the presence of interactions with phonons or other excitations. This interaction leads to phonon assisted resonant tunneling [1], and affects the peak-to-valley current ratio, which is important in device applications. Most previous treatments of this problem use a Green's function approach, often involving Keldysh formalism [2] 9]. An exact solution was obtained only for the special case of a single site coupled to a single phonon mode in $1 \mathrm{~d}$ [ 3 [ 6 ]. Experimentally important transverse degrees of freedom were treated only to leading order in perturbation theory [7].

The approach we use is to map the many-body problem in a possibly large variational space exactly onto a one-body problem. The Schrödinger equation, for the most part in real space, is then solved for the one-body problem. In our view, this is a conceptually simpler approach. It also produces explicit solutions for broad classes of problems that have not been solved before. The solution is essentially exact, in that the size of the variational space can be systematically increased until the answer converges. This approach need not make explicit use of Green's functions. (If desired, the Green's function can be recovered from the wavefunction.) Other inelastic tunneling problems can be solved by the same method.

One can solve essentially any problem where a single electron tunnels, and where manybody interactions are limited to a finite region of space. The electron-phonon coupling may be nonzero on many distinct sites, including several sites in the quantum well, sites in the barrier, and sites in the leads near the barrier. The electron may couple diagonally or offdiagonally to many types of phonons, and multiple quanta can be excited in the same or different modes. The electron-phonon coupling may be nonlinear, and the phonons may have nonlinear interactions among themselves. The electron can have transverse degrees of freedom. Arbitrary one-body interactions, including barriers and disorder, can also be included. The electron can interact in an arbitrary way, including spin-flip scattering, with a group of interacting "captive" electrons in the tunneling region, so long as the captive electrons cannot escape into the leads. The method can also be used at non-zero temperature. In practice, the method may make significant demands on computer resources when 
more than about 10,000 inelastic channels are included.

We consider the Hamiltonian

$$
\begin{aligned}
H & =H_{e l}+H_{p h}+H_{e l-p h}, \\
H_{e l} & =\sum_{j} \epsilon_{j} c_{j}^{\dagger} c_{j}-\sum_{j, k} t_{j, k}\left(c_{j}^{\dagger} c_{k}+h . c .\right), \\
H_{p h} & =\sum_{m} \omega_{m} a_{m}^{\dagger} a_{m}, \\
H_{e l-p h} & =-\sum_{j, m} \lambda_{j, m} c_{j}^{\dagger} c_{j}\left(a_{m}^{\dagger}+a_{m}\right) .
\end{aligned}
$$

The potential $\epsilon_{j}$ on site $j$ can describe a tunnel barrier, disorder, or a bias voltage. The hopping amplitude $t_{j, k}$ can vary from site to site. $\lambda_{j, m}$ is the (diagonal) coupling of an electron on site $j$ to an optical or acoustic phonon mode $m$. A site can represent a single atomic Wannier orbital or a larger region of space [10].

The method works for complicated barrier structures and interactions described by the above Hamiltonian, and for more general Hamiltonians. To illustrate the method in a simple context, however, we first consider the case with a single phonon mode that couples only to the electron density on site 0 . The many-body problem is first restricted to a variational subspace. For illustrative purposes, only states containing 0, 1, or 2 phonon quanta are retained in the example. (A workstation could easily handle thousands of states.) The many-body scattering problem in the variational subspace is then mapped exactly onto a 1-body problem with many channels, as shown in Fig. (1)a) and explained in the caption. At zero temperature, an electron incident from the left is an incoming plane wave on the lower left lead. It has an amplitude to exit on any of the six leads, corresponding to elastic and inelastic backscattering and transmission.

We seek the solution of the Schrödinger equation $E \psi_{j}=\sum_{k} H_{j, k} \psi_{k}$ on the tight-binding lattice of Fig. (11a) with the known eigenvalue $E=-2 t \cos \left(k_{0}\right)+\epsilon$, where $t$ and $\epsilon$ are the hopping amplitude and diagonal energy of the left lead, and $k_{0}$ is the incoming wavevector. (The term "lead" refers to the translation invariant part of the system.) The boundary conditions are that an incoming wave is allowed on only one lead. The scattering problem is straightforward to solve. One method is to "prune" any lead that has only an outgoing 
wave. This exact procedure removes the lead from the problem, while changing the site energy $\epsilon_{j}$ on the last retained site to a value that is in general complex and changes with $E$. For example, the Schrödinger equation on site 0 is $E \psi_{0}=h_{1}+\epsilon_{0} \psi_{0}-t_{0,1} \psi_{1}$, where $h_{1}$ includes the off-diagonal matrix elements from site 0 to sites other than 1 . To prune the lower right lead in Fig. (仿), note that there is a unique outgoing wave of energy $E$ in the lead, $\psi_{j}=A \exp \left(i k_{1} j\right)$. (If there are no propagating modes, choose instead the decaying mode $\psi_{j}=A \exp \left(-q_{1} j\right)$.) Back propagate this solution (through tunnel barriers if necessary) to obtain $\psi_{0}$ and $\psi_{1}$, using the Schrödinger equation on each site $j$ to obtain $\psi_{j-1}$ as a function of $\psi_{j}$ and $\psi_{j+1}$. The new Schrödinger equation on site 0, with all the sites in the lead removed, is now $E \psi_{0}=h_{1}+\tilde{\epsilon}_{0} \psi_{0}$, where $\tilde{\epsilon}_{0}=\epsilon_{0}-\alpha t_{0,1}$, and $\alpha \equiv \psi_{1} / \psi_{0}$ is generally complex. The leads to be pruned in Fig. (17a) are marked with vertical lines. The system to be solved after pruning is shown in Fig. (11b).

The problem in Fig. (11 b) is so simple that it is best solved by a recursive trick, which does not work in general. To motivate the general solution, consider the same problem, but where the electron at site 0 interacts with two distinct phonon modes of different frequencies. Again for illustrative purposes, choose a variational space that allows up to 2 phonon quanta in either mode, for a total of $3 \times 3=9$ phonon states (see Fig. Пc).

The pruned problem is not solved as a standard eigensystem, since the eigenvalue $E$ is known in advance. Considering the amplitude $\psi_{0}$ to be known, the problem is then to solve a system of (complex) linear equations of the form $A x=b$, where $x$ and $b$ are vectors, with $b$ proportional to $\psi_{0}$. For this toy problem $A$ is an $8 \times 8$ matrix. Once the system $A x=b$ is solved, the Schrödinger equation on sites 0, -1, etc., is used to determine the wavefunction on the first two sites of the left lead, and thus the coefficients $a_{1}$ and $a_{2}$ in $\psi_{j}=a_{1} \exp \left(i k_{0} j\right)+a_{2} \exp \left(-i k_{0} j\right)$. The current $J$ leaving through the pruned leads, corresponding to elastic or inelastic transmission or backscattering in particular channels, is obtained using $J_{j \rightarrow k}=2 \operatorname{Im}\left(\psi_{k}^{*} t_{k, j} \psi_{j}\right)$. The formula is applied for a retained site $j$ and a neighboring pruned site $k$. Current is conserved exactly, globally and at each vertex. This equation can be used to calculate ordinary current or a generalized current between two 
many-body states [11].

Another form of electron-phonon coupling modulates the hopping matrix element $t$ rather than the on-site energy $\epsilon$,

$$
H_{e l-p h}^{\prime}=-\sum_{j, k, m} \gamma_{j, k, m} c_{j}^{\dagger} c_{k}\left(a_{m}^{\dagger}+a_{m}\right)+h . c .
$$

This off-diagonal coupling represents the fact that when an atom is displaced to the right, the hopping amplitude $t$ to the atom on its right increases, because it is closer. A system with both types of electron-phonon coupling, which can be solved by the same method, is shown in Fig. (11/d).

Figures (2-4) show essentially exact results for problems that to our knowledge have not been previously solved. Figure (2) plots transmission for more than one site coupled to a single phonon mode, and considers off-diagonal electron-phonon coupling. Figure (3) has coupling to many distinct phonon modes of different frequencies [12]. Finally, Fig. (四) considers transverse degrees of freedom with electron recoil, which was previously treated only to leading order in perturbation theory [7].

As a simple test case, Fig. (22) shows transmission through a quantum dot where a single phonon mode couples to an electron on a site 0 with electron-phonon coupling strength $\lambda$. Hopping matrix elements are $t_{k, l}=t_{0}$ between site 0 and sites \pm 1 and $t_{k, l}=t$ for other nearest neighbors. We model the weak coupling through a tunnel barrier by a reduced $t_{0}$ in this paper, although we could have just as easily used sites with increased $\epsilon_{j}$. The same phonon mode also modulates the hopping matrix element (see Eq. (5)). A variational space with up to 8 phonon quanta gives results accurate to the width of the plot lines. The inset of Fig. (2) shows the transmission for the case where phonons couple only to the electron density, i.e. $\gamma=0$. Our result agrees with previous calculations [6]. The one-phonon sideband that we calculate was first seen experimentally by Goldman et al. [1]. Note that the low-energy "elastic" peak and the one-phonon sideband (at $\omega \approx \omega_{0}-\lambda^{2} / \omega_{0}$ ) are each composed of both elastic and inelastic transmission. This is because there is an amplitude for the electron in the left lead to couple to the first excited state of the displaced harmonic 
oscillator for the electron on site 0 , and then to tunnel into the right lead annihilating the phonon excitation, leaving the (now undisplaced) harmonic oscillator in its ground state. The calculation describes both inelastic tunneling, where phonons are emitted, and polaron physics, where phonons are emitted and reabsorbed. Elastic and inelastic tunneling can be experimentally distinguished by electroluminescence measurements [13].

Figures (2a-d) show transmission when both diagonal and off-diagonal electron-phonon coupling is present. $\gamma_{\{L, R\}}$ modulates the hopping between site 0 and the $\{$ left,right $\}$ lead. The only difference between figures (2a-d) is the relative sign of the coupling constants. There are clearly dramatic interference effects, which occur generically when a phonon is coupled to more than one site. The transmission peaks are much wider in Fig. (22a) than in Fig. (2) $\mathrm{b}$ ). This is attributed to the fact that the line width is proportional to $\tilde{t}_{0}^{2}$, where the effective coupling $\tilde{t}_{0} \approx t_{0}+\gamma\langle x\rangle$. The oscillator acquires a positive displacement $\langle x\rangle$ due to the $\lambda$ coupling. Figures (2/ $)$ and Fig. (2d) represent the same physical system reversed left to right. The elastic part of the transmission is identical for the two cases, while the total transmission is quite different. This behavior is consistent with the unitarity requirements for a time-reversal invariant system with inelastic channels. The total integrated transmission for (22c) is quite different from (22d), because the sum rule [3] is strongly violated for $\gamma$-type electron-phonon coupling.

Figure (3) shows the transmission through a single site quantum dot, which is coupled to 10 phonon modes with different frequencies. The electron-phonon coupling is diagonal, as in Eq. (4). We compute transmission using variational spaces that contain up to a total $N_{p h}=1, \ldots, 4$ phonon quanta distributed in any way among the 10 modes. Using subroutines for large sparse systems [14], the system with $N_{p h}=4$ consisting of $N_{s t}=1000$ states (2000 channels, including transmission and reflection) requires 20 seconds of CPU time per energy point on a Sparc 10 workstation. Each frequency point requires the solution of a $N_{s t} \times N_{s t}$ sparse system of complex linear equations. The inset of Fig. (3i) displays an enlarged portion of the transmission function in the region of predominantly one-phonon contribution. The weak electron-phonon coupling $\lambda=0.5$ is in the experimentally relevant 
regime, where the single phonon peaks dominate those due to multi-phonons. Even so, it is clear that one does not get an accurate description of inelastic tunneling in a variational space containing only single phonon excitations, and that $N_{p h}=3$ is required to achieve reasonable convergence.

It is straightforward to include transverse degrees of freedom, for example to model the case where the tunnel barrier is an extended, perhaps planar structure. We investigated a model containing $N_{y}$ parallel leads with periodic boundary conditions in the (transverse) $y$-direction. The Hamiltonian, written with real-space indices $j, l$ in the $x$-direction and momentum space indices $k, q$ in the $y$-direction is

$$
\begin{aligned}
H_{t r} & =\sum_{j, k}\left(\epsilon_{j}-2 t_{y j} \cos k\right) c_{j, k}^{\dagger} c_{j, k}-\sum_{j, l, k} t_{j, l}\left(c_{j, k}^{\dagger} c_{l, k}+h . c .\right) \\
& +\sum_{k} \omega_{k} a_{k}^{\dagger} a_{k}-\lambda / \sqrt{N_{y}} \sum_{k, q} c_{0, k}^{\dagger} c_{0, k+q}\left(a_{q}^{\dagger}+a_{-q}\right),
\end{aligned}
$$

where the on-site energies are $\epsilon_{j}=\left[\epsilon_{l}, \epsilon_{0}, \epsilon_{r}\right]$ for $[j<0, j=0, j>0]$ respectively. Hopping matrix elements are $t_{j, l}=t_{x}$ for nearest neighbor $j, l \neq 0$, and $t_{j, l}=t_{x 0}$ when $j=0$ or $l=0$. Similarly $t_{y j}=t_{y}$ for $j \neq 0$ and $t_{y j}=t_{y 0}$ otherwise. Diagonal electron-phonon coupling is restricted to sites where $j=0$. Due to the translational symmetry in the $y$-direction, the total transverse momentum $k$ is conserved. The electron momentum changes only as a consequence of the electron-phonon interaction.

Figure (4) shows the transmission for $N_{y}=6$ parallel leads. Figure (4) uses a variational space with up to $N_{p h}=5$ phonon quanta in any phonon modes, which gives satisfactory accuracy in the whole frequency and transverse momentum range. For simplicity we use a dispersionless phonon spectrum; however, generalization to momentum-dependent phonon frequencies and electron-phonon coupling is straightforward. At small transverse momentum $k=0$ and $\pi / 3$, a strong nearly elastic resonance is located just below the noninteracting resonance at $\omega(k)=\epsilon_{0}-2 t_{y 0} \cos k$. Inelastic side-peaks at higher $\omega$ correspond to phonon creation, usually accompanied by a change in the electron momentum. For large transverse momentum $k=2 \pi / 3$ or $\pi$, resonant states where the electron creates a phonon and recoils to lower momentum can have a lower energy than the state with no phonons. This results 
in side-peaks at energies below the weakened central peak.

Generalizations: Finite temperature problems can be solved by having the incoming electron arrive on different leads in Fig. (11a) with the appropriate Boltzmann weights. It should now be possible to model more realistic coupled electron-phonon systems, to address such questions as why the inelastic peak is observed experimentally at the barrier phonon frequency rather than that of the well [1,15. It would be interesting to study the stronger electron-phonon coupling that arises when electrons are localized on impurities, or from phonon modes caused by crystal defects. It should also be possible to model more complicated band structures that include several Wannier functions per unit cell, and amorphous barriers if a suitable tight-binding description is known.

Acknowledgments: We thank Jim Gubernatis, Selman Hershfield, Alan Katz, Roger Lake, Steve Lyon, David Rabson, Darryl Smith, and John Wilkins for valuable discussions. This work was supported by the US DOE. 


\section{REFERENCES}

[1] V. J. Goldman, D. C. Tsui, and J. E. Cunningham, Phys. Rev. B 36, 7635 (1987).

[2] J. Kirtley, D. J. Scalapino, and P. K. Hansma, Phys. Rev. B 14, 3177 (1976).

[3] N. S. Wingreen, K. W. Jacobsen, and J. W. Wilkins, Phys. Rev. Lett. 61, 1396 (1988); P. Hyldgaard et al., Ann. Phys. 236, 1 (1994).

[4] L. I. Glazman and R. I. Shekhter, Sov. Phys. JETP 67, 163 (1988).

[5] B. Y. Gelfand et al., Phys. Rev. Lett. 62, 1683 (1989); W. Cai et al., Phys. Rev. Lett. 63, 418 (1989).

[6] J. A. Støveng et al., Phys. Rev. B 44, 13595 (1991).

[7] N. Zou and K.A. Chao, Phy. Rev. Lett. 69, 3224 (1992); N. Zou et al., Phys. Rev. B 46, 15912 (1992).

[8] E. V. Anda and F. Flores, J. Phys. Condens. Matter 3, 9087 (1991).

[9] R. Lake and S. Datta, Phys. Rev. B 45, 6670 (1992).

[10] Nonlinear phonon modes or nonlinear interactions among phonons can be modeled by adding terms of higher than quadratic order to $H_{p h}$, such as $\left(a^{\dagger}\right)^{4}+$ h.c. Nonlinear electron-phonon coupling could be included with terms such as $c^{\dagger} c\left(a^{\dagger}\right)^{2}+h . c$. The same method works with nonlinear terms included.

[11] Once the many-body problem has been mapped onto a one-body problem as shown in Fig. (11a), the reader who prefers can use Green's function methods at this point, rather than solving for the wavefunction. The change in energy $-\alpha t_{0,1}$ is then the self energy.

[12] A single site coupled to many phonons of the same frequency has been considered previously. For identical frequencies, a suitable linear combination of phonon coordinates results in coupling to a single phonon mode, and $n-1$ decoupled modes. This simplification does not occur for phonon modes of different frequencies. 
[13] R. Teissier et al., Phys. Rev. B 50, 4885 (1994).

[14] Z. Zlatev et al., Computer Science, Y12M Solution of Large and Sparse Systems of Linear Algebraic Equations, Documentation of Subroutines, ed. G. Goos and J. Hartmanis (Springer-Verlag, 1981).

[15] N. Mori and T. Ando, Phys. Rev. B 40, 6175 (1989); P. J. Turley and S. W. Teitsworth, Phys. Rev. B 44, 8181 (1991). 


\section{FIGURES}

FIG. 1. (a) Each dot represents a basis state $|j, n\rangle$ in the many-body Hilbert space. The rows of dots are the sites $j=-3, \ldots, 3$ with $n=0,1$, or 2 phonon quanta. The bonds represent non-zero off-diagonal matrix elements in the Hamiltonian. The horizontal bonds are the hopping amplitudes $t_{j, k}$. The vertical bonds represent the electron-phonon interaction, with amplitudes $-\lambda$ on the lower and $-\lambda \sqrt{2}$ on the upper vertical bond. The dots can also be interpreted as Wannier orbitals in an equivalent 1-body tight-binding model. (b) The system that results after pruning all but the incoming (lower left) lead. Sites with complex diagonal energies $\epsilon_{j}$ are shaded gray. (c) The pruned version of the problem where an electron at site 0 couples to two distinct phonon modes. In the $3 \times 3$ grid of gray dots, the vertical direction is the number of quanta $n_{1}=0,1,2$ of type 1 phonons, and the direction into the page is the number of quanta $n_{2}$ of type 2 phonons. Before pruning, there was one right and one left lead attached to each gray dot. (d) A system with a single phonon mode, diagonal electron-phonon coupling of the type in Eq. (41) on site 0, and off-diagonal electron-phonon coupling of the type in Eq. (5) between site 0 and sites \pm 1 . The pruned version is shown.

FIG. 2. Transmission probability as a function of the incident electron energy. The heavy line is the total transmission, and the lighter (lower) line the elastic part. A single phonon mode is coupled to the electron density at site 0 with strength $\lambda$, and to the electron hopping amplitude between site 0 and sites \pm 1 with strength $\gamma$. The other parameters of the Hamiltonian are: $\epsilon_{l}=\epsilon_{r}=\epsilon_{0}=0.0$ (no voltage drop across the dot), $t_{0}=0.2, \omega_{0}=1.0$ and $N_{p h}=8$. All energies are in units of the hopping $t$ in the leads.

FIG. 3. Transmission through a single site that is coupled to ten phonon modes with phonon frequencies $\omega_{n}$ uniformly distributed between $\omega_{\min }$ and $\omega_{\max }$, with electron-phonon coupling constants $\lambda_{n}=0.5 \omega_{n}$. Different curves represent runs with basis sets containing a different total number of phonon quanta $N_{p h}$. 
FIG. 4. Transmission through $N_{y}=6$ transverse channels as a function of the energy $\omega$ of the incident electron at four different choices of transverse momentum $k$, with $t_{x 0}=0.2$. The upper and lower lines represent total and elastic transmission respectively. 


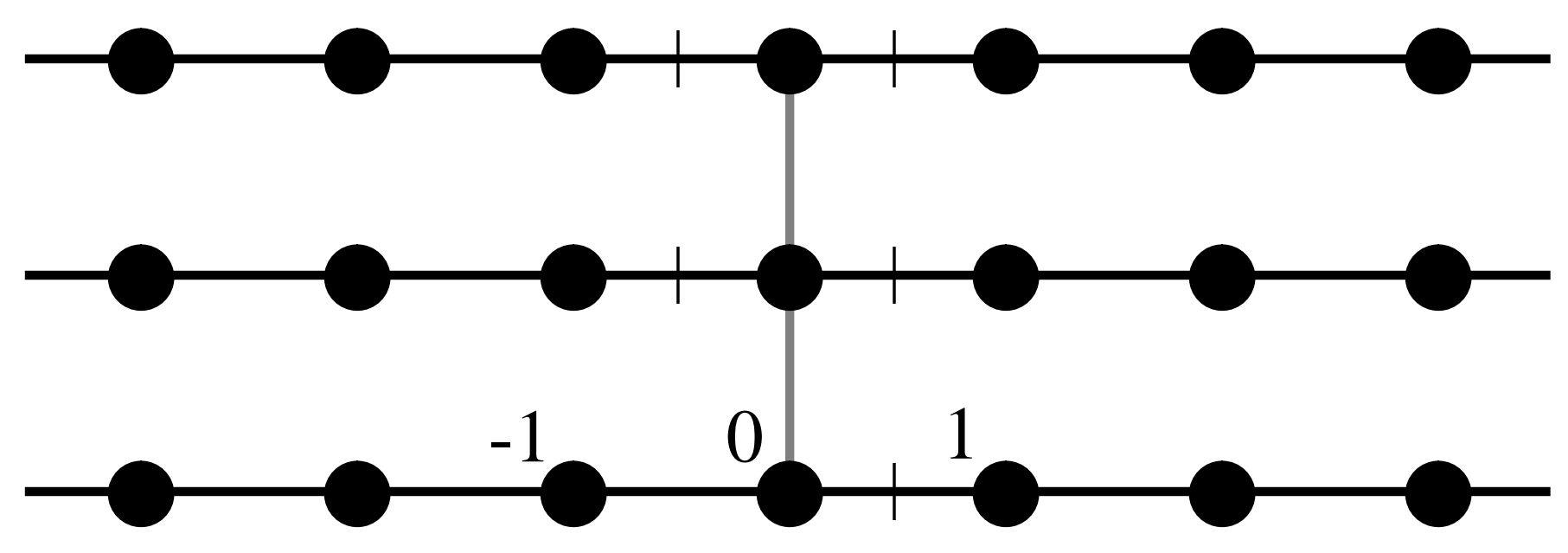

(a)
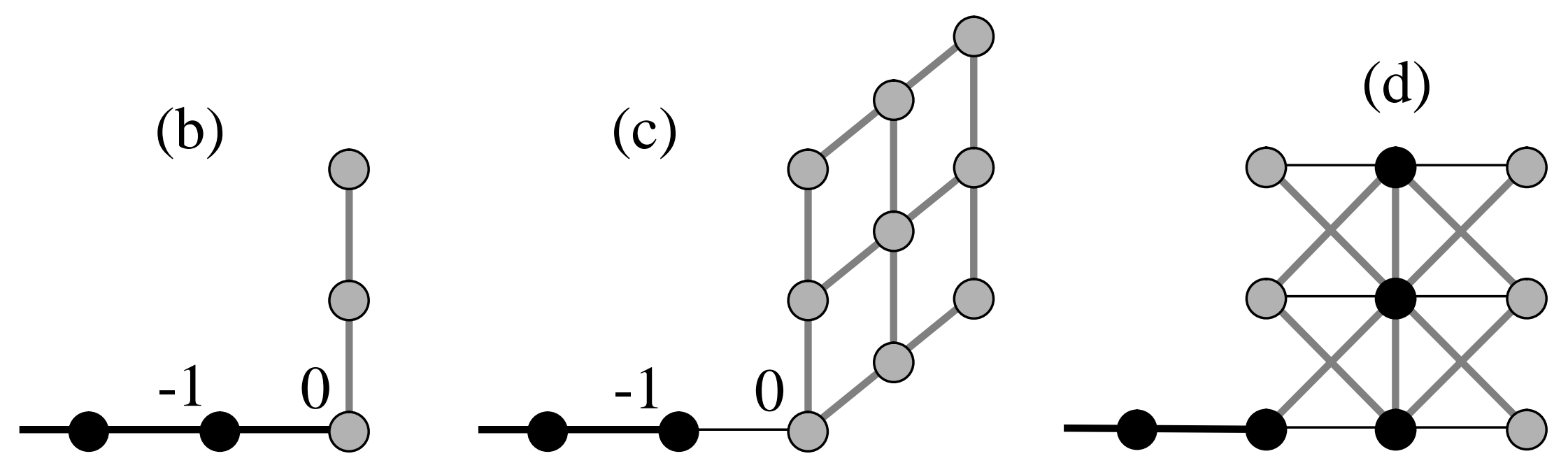


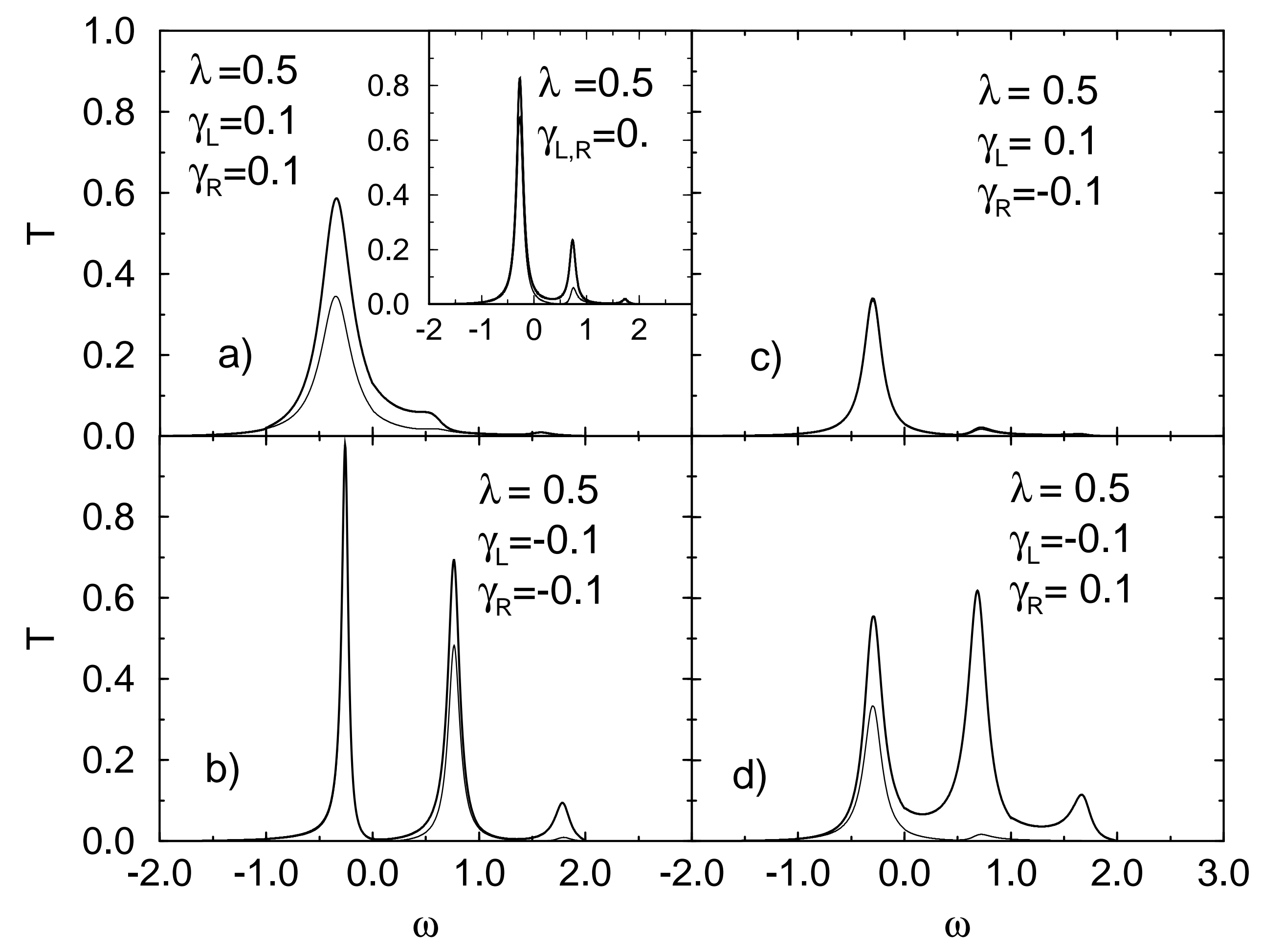




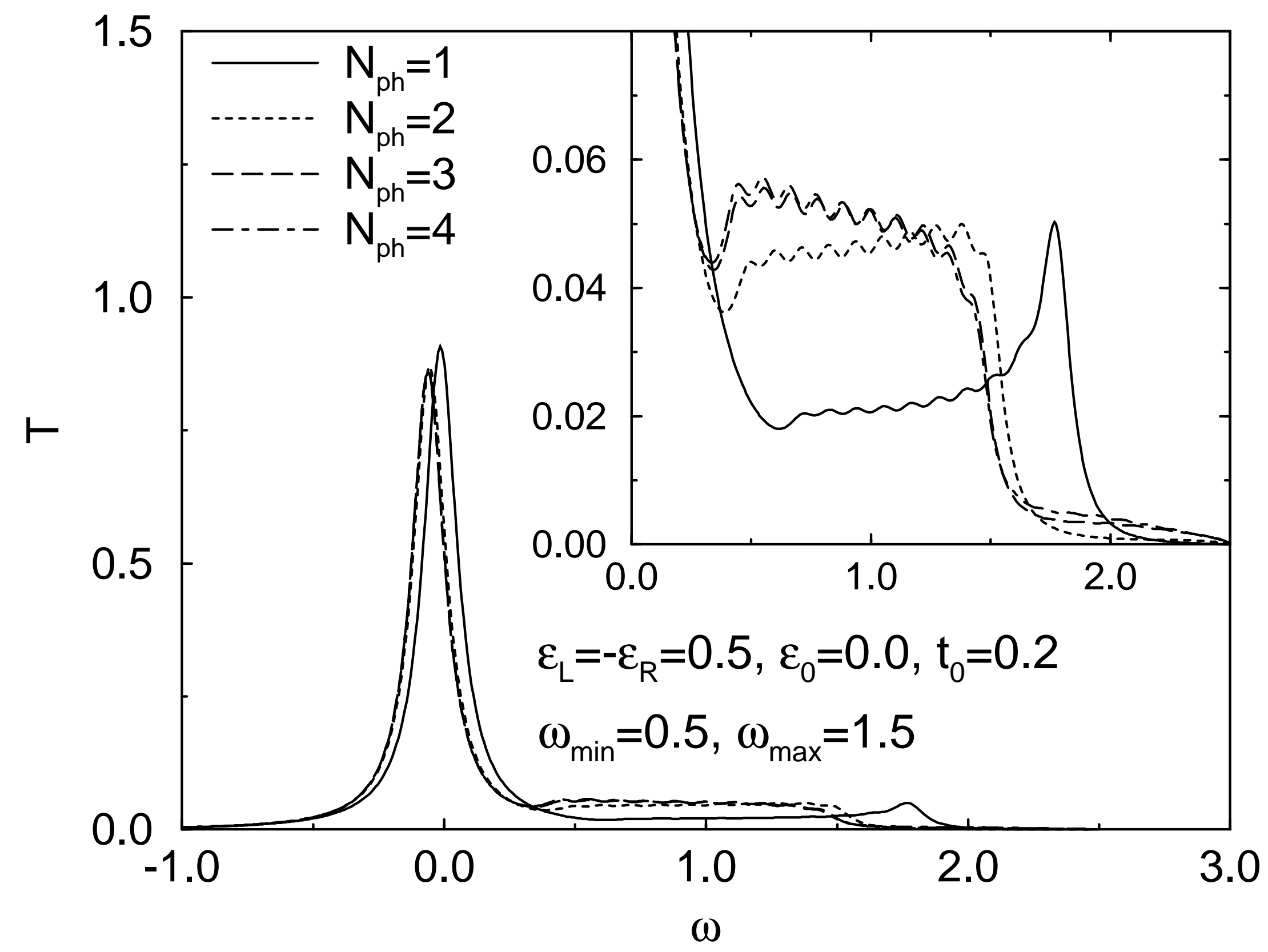




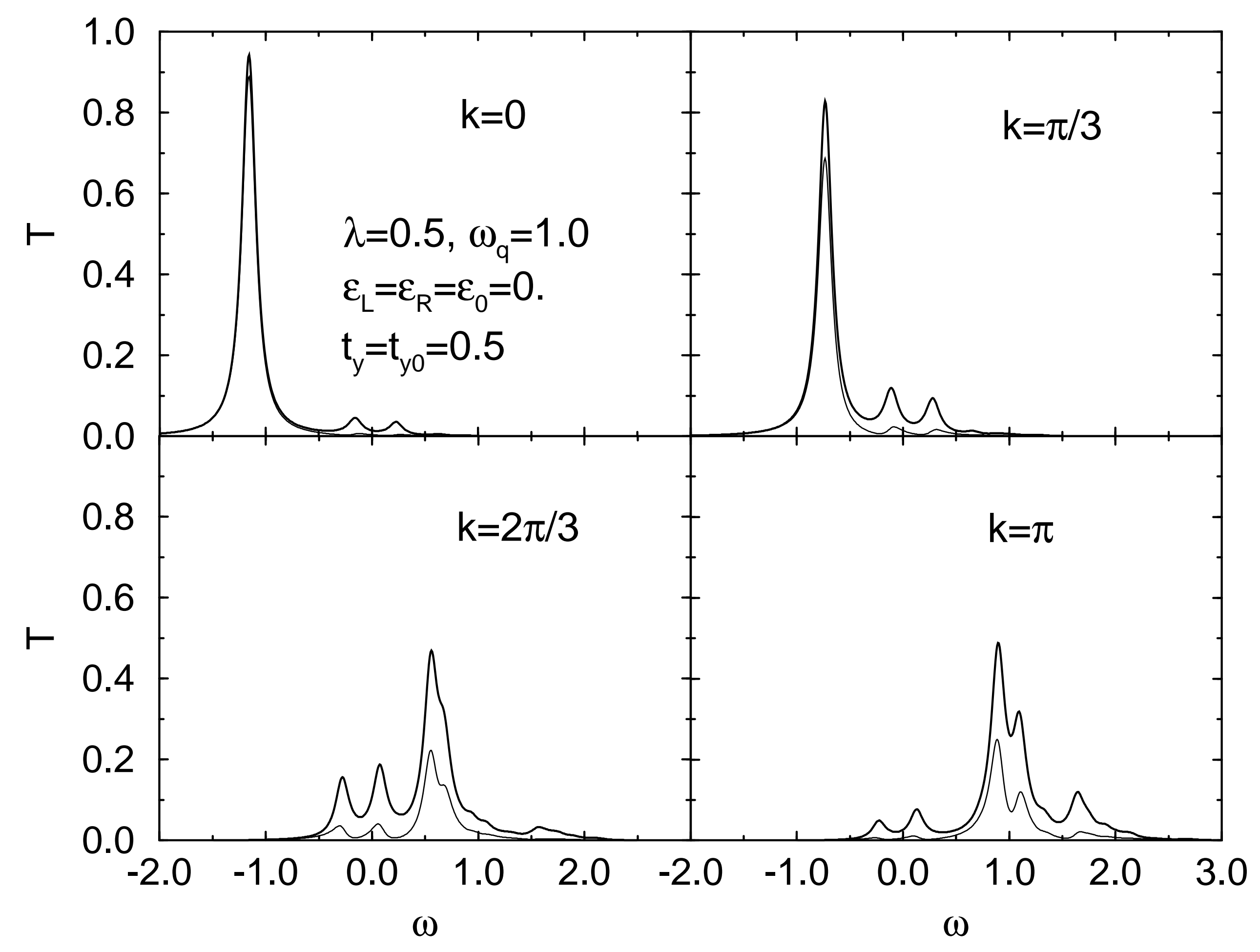

